\section{(1)}

CrossMark

\title{
Pulmonary hypertension after bone marrow transplantation in children
}

\author{
Marilyne Levy ${ }^{1,2,3}$, Despina Moshous ${ }^{1,4,5}$, Isabelle Szezepanski², \\ Louise Galmiche ${ }^{6}$, Martin Castelle ${ }^{1,4}$, Fabrice Lesage ${ }^{1,7}$, Laurent Dupic ${ }^{1,7}$, \\ Bénédicte Neven ${ }^{1,4,5}$, Alain Fischer ${ }^{1,4,5,8}$, Stéphane Blanche ${ }^{1,4}$ and \\ Damien Bonnet ${ }^{1,2}$
}

Affiliations: ${ }^{1}$ Université Paris Descartes, Sorbonne Paris Cité, Paris, France. ${ }^{2} \mathrm{M} 3 \mathrm{C}$-Unité Médico-Chirurgicale de Cardiologie Pédiatrique, Hôpital Necker-Enfants Malades, AP-HP, Paris, France. ${ }^{3}$ UE3C-Unité d'Explorations Cardiologiques-Cardiopathies Congénitales, Paris, France. ${ }^{4}$ Pediatric HaematologyImmunology and Rheumatology Unit, Hôpital Necker-Enfants Malades, AP-HP, Paris, France. ${ }^{5}$ INSERM U1163 and Institut Imagine, Paris, France. 'Service d'Anatomopathologie, Hôpital Necker-Enfants Malades, Paris, France. ${ }^{7}$ Pediatric Intensive Care Unit, Hôpital Necker-Enfants Malades, AP-HP, Paris, France. ${ }^{8}$ Collège de France, Paris, France.

Correspondence: Marilyne Levy, M3C-Unité Médico-Chirugicale de Cardiologie Pédiatrique, Hôpital NeckerEnfants Malades, 149 Rue de Sèvres, 75015 Paris, France. E-mail: marilyne.levydue3c.fr

@ERSpublications

Pulmonary hypertension is a rare and severe complication of haematopoietic stem cell transplantation in children, with a high mortality if misdiagnosed. Early diagnosis and treatment allow rapid clinical improvement with normalisation of PAP in most cases. http://bit.ly/2KXyhNN

Cite this article as: Levy M, Moshous D, Szezepanski I, et al. Pulmonary hypertension after bone marrow transplantation in children. Eur Respir J 2019; 54: 1900612 [https://doi.org/10.1183/13993003.00612-2019].

\section{ABSTRACT}

Introduction: Pulmonary hypertension is a rare but important cause of mortality after haematopoietic stem cell transplantation (HSCT) in children. This complication is poorly characterised in the literature. We report here a series of children who developed pulmonary hypertension after HSCT.

Methods: Between January 2008 and December 2015, we retrospectively analysed 366 children who underwent HSCT (age range 0.5-252 months; median 20.3 months). During the post-HSCT course, echocardiography scans motivated by respiratory symptoms identified 31 patients with elevated tricuspid regurgitation velocity $\left(>2.8 \mathrm{~m} \cdot \mathrm{s}^{-1}\right)$, confirmed when possible by right heart catheterisation (RHC).

Results: 22 patients had confirmed pulmonary hypertension with mean \pm SD pulmonary arterial pressure $40.1 \pm 10 \mathrm{mmHg}$ (range $28-62 \mathrm{mmHg}$ ) and pulmonary vascular resistance 17.3 \pm 9.2 Wood Units (range 842 Wood Units). Among the 13 responders at reactivity test, only one patient responded to calcium channel blockers. Seven patients (32\%) died. 15 pulmonary hypertension patients were alive after a mean \pm sD follow-up of $6.5 \pm 2.3$ years (range 2-10 years). All survivors could be weaned off pulmonary hypertension treatment after a median follow-up of 5 months (range 3-16). The delay between clinical symptoms and initiation of pulmonary hypertension therapy was significantly longer in patients who subsequently died (mean \pm SD $33.5 \pm 23$ days; median 30 days) than in survivors (mean \pm SD $7 \pm 3$ days) $(\mathrm{p}<0.001)$.

Conclusion: Pulmonary hypertension is a severe complication of HSCT with an underestimated incidence and high mortality. Aggressive and timely up-front combination therapy allowed normalisation of pulmonary pressure and improved survival. 


\section{Introduction}

Pulmonary arterial hypertension (PAH) is a rare but serious complication of haematopoietic stem cell transplantation (HSCT) with a fatal outcome in the majority of reported cases $[1,2]$. Since the first case report of fatal pulmonary hypertension in a 12-year-old boy who underwent HSCT for acute lymphoblastic leukaemia in 1984 by TROUSSARD et al. [3], a number of single-patient case reports and only a few small case series have been published [4-7]. The incidence of pulmonary hypertension after HSCT has been estimated at between $15 \%$ and $28 \%$. Pulmonary hypertension after HSCT was surprisingly frequent in malignant osteopetrosis, suggesting that the underlying disease could be a risk factor for developing pulmonary hypertension after HSCT $[4,6]$. The most commonly reported pathological types of pulmonary hypertension are pulmonary arterial hypertension $(\mathrm{PAH})$ and pulmonary veno-occlusive disease (PVOD). The prognosis appears to be poor. In addition, the risk factors for developing pulmonary hypertension after HSCT and the predictors of outcome are not known. Certainly, factors predisposing to pulmonary hypertension after HSCT as well as risk factors for mortality are multiple in this setting.

As the diagnosis of pulmonary hypertension after HSCT is challenging and the therapeutic strategy unclear, we retrospectively reviewed all cases of children referred for suspected pulmonary hypertension after HSCT in our institution and their outcomes.

\section{Patients and methods}

Between January 2008 and December 2015, all patients who developed unexplained respiratory symptoms after HSCT performed in the Paediatric Immunology Unit at Necker Children's Hospital (Paris, France) were referred to the Dept of Paediatric Cardiology for noninvasive evaluation of pulmonary pressure by echocardiography. If tricuspid regurgitation velocity (TRV) was $<2.8 \mathrm{~m} \cdot \mathrm{s}^{-1}$, pulmonary hypertension was considered unlikely in the absence of additional echocardiographic variables suggestive of pulmonary hypertension [8]. If TRV was $\geqslant 2.8 \mathrm{~m} \cdot \mathrm{s}^{-1}$ with or without additional variables suggestive of pulmonary hypertension, right heart catheterisation (RHC) was performed to confirm pulmonary hypertension whenever the child's clinical status allowed this procedure. Diagnosis of pulmonary hypertension was confirmed if mean pulmonary arterial pressure (mPAP) $\geqslant 21 \mathrm{mmHg}$; measurements were repeated in the presence of oxygen and nitric oxide in order to test pulmonary vasoreactivity. According to the Sitbon criteria, patients were considered responders if during catheterisation, mPAP decreased by $20 \%$ and reached a level $<40 \mathrm{mmHg}[8,9]$.

Whenever pulmonary hypertension was confirmed, targeted PAH treatments were introduced in addition to supportive therapy including oxygen. The treatment was decided according to the clinical and haemodynamic status at diagnosis. Patients who were considered responders during the vasoreactivity test received calcium channel blockers (CCBs) only if they were in stable haemodynamic condition. Nonresponders received either monotherapy (phosphodiesterase-5 inhibitor (PDE5i) or endothelin receptor antagonist (ERA)), oral bitherapy (PDE5i and ERA) or triple therapy with PDE5i, ERA and prostanoids $(n=4)$. Monitoring of pulmonary hypertension and right heart function was performed sequentially after treatment initiation. N-terminal pro-brain natriuretic peptide and circulating endothelial cells were measured in a limited number of patients at baseline and during follow-up.

Lung biopsy was performed in three patients (post-mortem in two of them). In addition to the usual stainings, immunostaining was performed for CD34 (M7165, 1/50; Dako, Les Ulis, France), smooth muscle actin (M0851, 1/500; Dako), caldesmon (M3557, 1/200; Dako), CD45 (M0701, 1/200; Dako), CD3 (A0452, 1/200; Dako), CD20 (M0755, 1/400; Dako) and CD68 (M0814, 1/3000; Dako) antibodies.

During the study period, a total of 366 HSCT procedures were performed in the Paediatric Immunology Unit at Necker Children's Hospital, including 27 patients requiring a second HSCT and six patients requiring a third HSCT. 26 patients received a stem cell boost without conditioning. Indications for allogenic HSCT in patients who developed pulmonary hypertension were familial lymphohistiocytosis $(n=8)$, severe combined immunodeficiency (SCID) $(n=3)$, osteopetrosis $(n=1)$, chronic granulomatous disease $(n=1)$, neonatal aplasia $(n=1)$, leaky SCID $(n=1)$ and Omenn syndrome $(n=3)$. The conditioning regimens are detailed in figure 1. Two-thirds of all HSCT patients with conditioning received defibrotide prophylactically, including all but one patient who developed pulmonary hypertension. Four additional patients were referred from other centres after HSCT for neuroblastoma $(n=3)$ and medulloblastoma $(n=1)$.

Written informed consent was obtained from the patients' parents regarding HSCT, anonymous data collection and analysis. This was in done accordance with the Declaration of Helsinki and French laws and regulations.

\section{Statistical analysis}

Descriptive data are expressed as mean $\pm \mathrm{SD}$ or median. Comparisons between groups for different treatments, delayed diagnosis or mortality were assessed using the nonparametric Mann-Whitney U-test, 


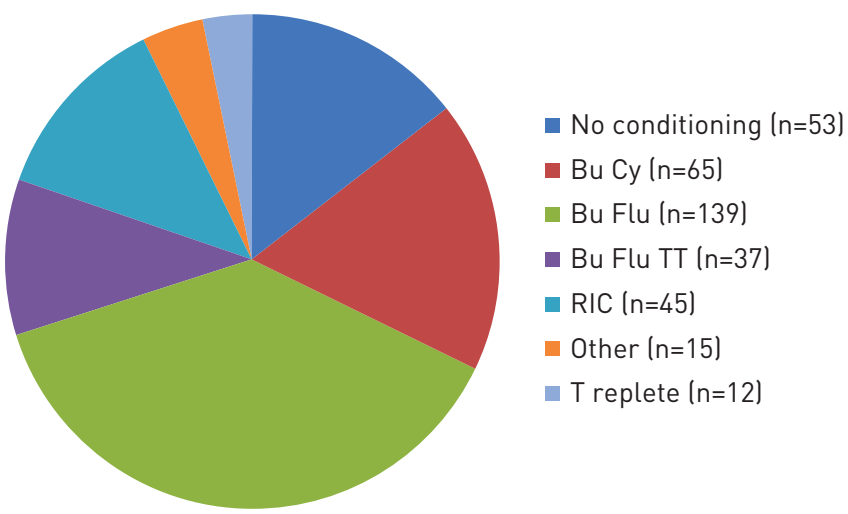

FIGURE 1 Conditioning regimens of the 366 children before haematopoietic stem cell transplantation (HSCT). Bu Cy: busulfan and cyclophosphamide (200 $\mathrm{mg} \cdot \mathrm{kg}^{-1}$ total dose); Bu Flu: busulfan and fludarabine; Bu Flu TT: busulfan, fludarabine and thiotepa; RIC: reduced intensity conditioning; T replete: busulfan, fludarabine and post-HSCT cyclophosphamide (100 $\mathrm{mg} \cdot \mathrm{kg}^{-1}$ total dose).

independent samples t-test or Fisher's test when appropriate. Statistical significance was inferred at a two-sided p-value $<0.05$.

\section{Results}

Symptoms of pulmonary hypertension after HSCT were respiratory in all patients, and included tachypnoea, hypoxia and respiratory distress resistant to usual symptomatic treatment including oxygen, antibiotics and corticosteroids. These symptoms occurred at a median of 56 days after HSCT (range 15120 days). The median age at HSCT of patients who developed pulmonary hypertension was 26 months (range 24-48 months) in the four patients with solid tumours, whereas in patients transplanted for nonmalignant inborn errors the median age at HSCT was 4.4 months (range $2.8-13.8$ months; $\mathrm{p}<0.01$ ) in patients who developed pulmonary hypertension compared with a median age of 20.3 months (range $0.5-$ 252.6 months) in the whole group.

Among the 70 patients with unexplained respiratory symptoms referred for noninvasive evaluation of pulmonary pressure, $31(42 \%)$ had TRV $>2.8 \mathrm{~m} \cdot \mathrm{s}^{-1}$ at echocardiography scan. Among theses 31 patients, five with high pulmonary pressure (TRV $>4 \mathrm{~m} \cdot \mathrm{s}^{-1}$ ) were too unstable to have haemodynamic evaluation and 26 underwent RHC. RHC showed normal pulmonary pressure in nine patients and confirmed pulmonary hypertension in 17 patients. Finally, 22 met the criteria for pulmonary hypertension: 17 confirmed by RHC and five by echocardiography (table 1 and figure 2). Among the 17 patients with RHC-confirmed pulmonary hypertension, 15 had reactivity testing and 13 out of 15 were considered responders according to the Sitbon criteria.

Out of 253 patients who received a conditioning regimen containing busulfan, 17 developed pulmonary hypertension, whereas no patient developed PAH out of 60 patients who received conditioning without busulfan (figure 3 ). This was found not significant $(\mathrm{p}=0.0508)$ by Fisher's exact test. Of note, one patient in the remaining group of 53 patients who did not receive any chemotherapy in the conditioning developed pulmonary hypertension. This patient had a severe lung infection pre-HSCT (human metapneumovirus and Pneumocystis jirovecii). However, the association of busulfan with fludarabine and thiotepa (Bu Flu TT), another alkylating agent, was significantly correlated to pulmonary hypertension $(\mathrm{p}=0.0054)$, whereas the use of busulfan associated to cyclophosphamide (Bu Cy) or fludarabine (Bu Flu) did not reach significance ( $\mathrm{p}=0.34$ and 0.81 , respectively).

12 patients developed hepatic veno-occlusive disease prior to pulmonary hypertension (66\%), whereas the incidence of veno-occlusive disease in all HSCT procedures was only $\sim 17 \%$. All but two pulmonary hypertension patients had received prophylactic defibrotide.

With regard to the underlying disease, in our series, the proportion of pulmonary hypertension was significantly greater in patients with lymphohistiocytosis $(\mathrm{p}=0.0042)$.

The time between the onset of respiratory symptoms and diagnosis of pulmonary hypertension was $17.5 \pm 18.7$ days. In the course of the observation period, there was an important shortening of the delay between first clinical symptoms and diagnosis as a consequence of increasing awareness. The delay was $22.2 \pm 20.9$ days before 2012 and $4.15 \pm 1.68$ days after $2012(\mathrm{p}<0.05)$. At that time we organised a specific protocol with repeated echocardiography in patients with clinical suspicion of pulmonary hypertension and haemodynamic evaluation to confirm it. 
TABLE 1 Characteristics of patients who developed pulmonary hypertension after haematopoietic stem cell transplantation (HSCT)

\begin{tabular}{|c|c|c|c|c|c|c|c|c|c|}
\hline Patient & Cause of HSCT & $\begin{array}{c}\text { Age at } \\
\text { HSCT } \\
\text { months }\end{array}$ & $\begin{array}{c}\text { Day of } \\
\text { pulmonary } \\
\text { hypertension } \\
\text { diagnosis }\end{array}$ & $\begin{array}{c}\text { Pulmonary } \\
\text { hypertension } \\
\text { symptoms } \\
\text { diagnosis delay } \\
\text { days }\end{array}$ & $\begin{array}{c}\text { NHYA } \\
\text { functional } \\
\text { class at } \\
\text { diagnosis }\end{array}$ & $\begin{array}{l}\text { mPAP } \\
\text { mmHg }\end{array}$ & $\begin{array}{l}\text { PVR } \\
\text { WU }\end{array}$ & Outcome & $\begin{array}{l}\text { Follow-up } \\
\text { delay years }\end{array}$ \\
\hline 1 & Lymphohistiocytosis & 4 & +90 & 3 & III & 53 & 12.5 & Alive & 10 \\
\hline 2 & Osteopetrosis & 4 & +60 & 5 & III & 32 & 13 & Alive & 5 \\
\hline 3 & CGD & 6 & +60 & 7 & IV & 28 & 12 & Alive & 3 \\
\hline 4 & Neuroblastoma & 26 & +150 & 70 & III & 31 & 8 & Died & \\
\hline 5 & Neonatal aplasia & 3 & +60 & 5 & III & 32 & 14 & Alive & 6 \\
\hline 6 & SCID & 10 & +30 & 2 & III & 31 & 15 & Alive & 5 \\
\hline 7 & Neuroblastoma & 48 & +60 & 30 & IV & 47 & 11.5 & Alive & 7 \\
\hline 8 & Lymphohistiocytosis & 4 & +60 & 20 & III & 52 & 31 & Alive & 6 \\
\hline 9 & SCID & 13 & +90 & 60 & IV & 43 & 15 & Died & \\
\hline 10 & Omenn syndrome & 3 & +90 & 12 & IV & 40 & 20 & Alive & 7 \\
\hline 11 & Medulloblastoma & 24 & +210 & 5 & IV & 31 & 5 & Alive & 10 \\
\hline 12 & Griscelli syndrome & 8 & +90 & 30 & V & 29 & 18 & Died $^{\#}$ & \\
\hline 13 & Lymphohistiocytosis & 3 & +60 & 4 & IV & 46 & 19 & Alive & 6 \\
\hline 14 & Lymphohistiocytosis & 4 & +90 & 3 & III & 28 & 27 & Alive & 5 \\
\hline 15 & Omenn syndrome & 3 & +60 & 20 & IV & 45 & NA & Alive & 9 \\
\hline 16 & Leaky SCID & 10 & +60 & 30 & IV & 48 & NA & Died & \\
\hline 17 & SCID & 3 & +60 & 3 & IV & 62 & 42 & Alive & 3 \\
\hline 18 & Omenn syndrome & 4 & +60 & 35 & IV & 50 & NA & Died & \\
\hline 19 & Lymphohistiocytosis & 5 & +60 & 4 & IV & 50 & NA & Alive & 9 \\
\hline 20 & Neuroblastoma & 26 & +80 & 5 & III & 46 & 14 & Alive & 7 \\
\hline 21 & Lymphohistiocytosis & 5 & +60 & 4 & IV & 22 & 4 & Died & \\
\hline 22 & Lymphohistiocytosis & 2.5 & +60 & 5 & IV & 56 & NA & Died & \\
\hline
\end{tabular}

NYHA: New York Heart Association; mPAP: mean pulmonary arterial pressure; PVR: pulmonary vascular resistance; WU: Wood Units; CGD: chronic granulomatous disease; SCID: severe combined immunodeficiency disease; NA: not available. ${ }^{\#}$ : died of infection.

Whenever pulmonary hypertension was confirmed by RHC $(n=17)$ or by echocardiography $(n=5)$, a specific treatment was initiated, except for one patient who died before treatment initiation (figure 4). From the 13 responders, all patients but one needed add-on or switch to ERA or PDE5i. The first-line treatment was decided according to the clinical and haemodynamic status at diagnosis. After 2012, treatment was escalated in the absence of rapid improvement at 24 or $48 \mathrm{~h}$ evaluation. The treatment in the 21 treated patients was monotherapy in 12 patients $(n=4$ CCB, $n=7$ PDE5i and $n=1$ ERA), oral combination therapy in five patients $(n=1$ CCB+PDE5i and $n=4$ ERA+PDE5i) and up-front triple therapy in four patients (ERA+PDE5i+prostanoids). Sequential add-on therapy was performed in seven patients who received initial monotherapy or oral combination.

The overall mortality in the HSCT patients was $23.5 \%$ (86 out of 366). From the 22 pulmonary hypertension patients, seven died $(31.8 \%$; $\mathrm{p}=0.3$ compared with patients without pulmonary hypertension patients): six from severe and progressive pulmonary hypertension and one from infection 6 months after normalisation of pulmonary pressure. 15 pulmonary hypertension patients were alive after a follow-up of $7.5 \pm 2.3$ years (range 3-11 years). All of them normalised their pulmonary pressure within $19 \pm 8$ days (range 15-40 days) and could be weaned off pulmonary hypertension treatment after $6.1 \pm 3.2$ months (median 5 months; range 3-16 months). In all 15 survivors, repeated echocardiography showed sustained normal pulmonary pressure with a follow-up of $7.5 \pm 2.3$ years (range 3-11 years). Of note, the delay between clinical symptoms and initiation of pulmonary hypertension therapy was significantly longer in patients who died ( $33.5 \pm 25$ days) than in survivors $(7 \pm 3$ days $)(p<0.001)$.

Lung biopsy was performed in three patients, showing preservation of global lung architecture. Vascular lesions associate with medial hypertrophy and intimal proliferation occluding the lumen in intra-acinar pulmonary arteries (figure 5). Oedema of the medial and intimal layers was observed, as well as vacuoles in some arteries. The intimal lesions were due to heterogeneous cellular proliferation of myofibroblasts, endothelial cells and macrophages. These cellular lesions indicated an acute process with proliferation of endothelial cells, but without any intimal fibrosis or antiapoptotic protein Bcl-2 expression as described in irreversible pulmonary hypertensive lesions [10]. There were no plexiform lesions, veno-occlusive lesions or microvascular clots in the three patients with lung biopsy. 
FIGURE 2 Flow diagram showing diagnosis and outcome of haematopoietic stem cell transplantation (HSCT) patients suspected of having pulmonary hypertension. RHC: right heart catheterisation. \#: patients too haemodynamically unstable to have $\mathrm{RHC} ;{ }^{n}: \mathrm{n}=13$ reactive pulmonary hypertension at vasodilative test.

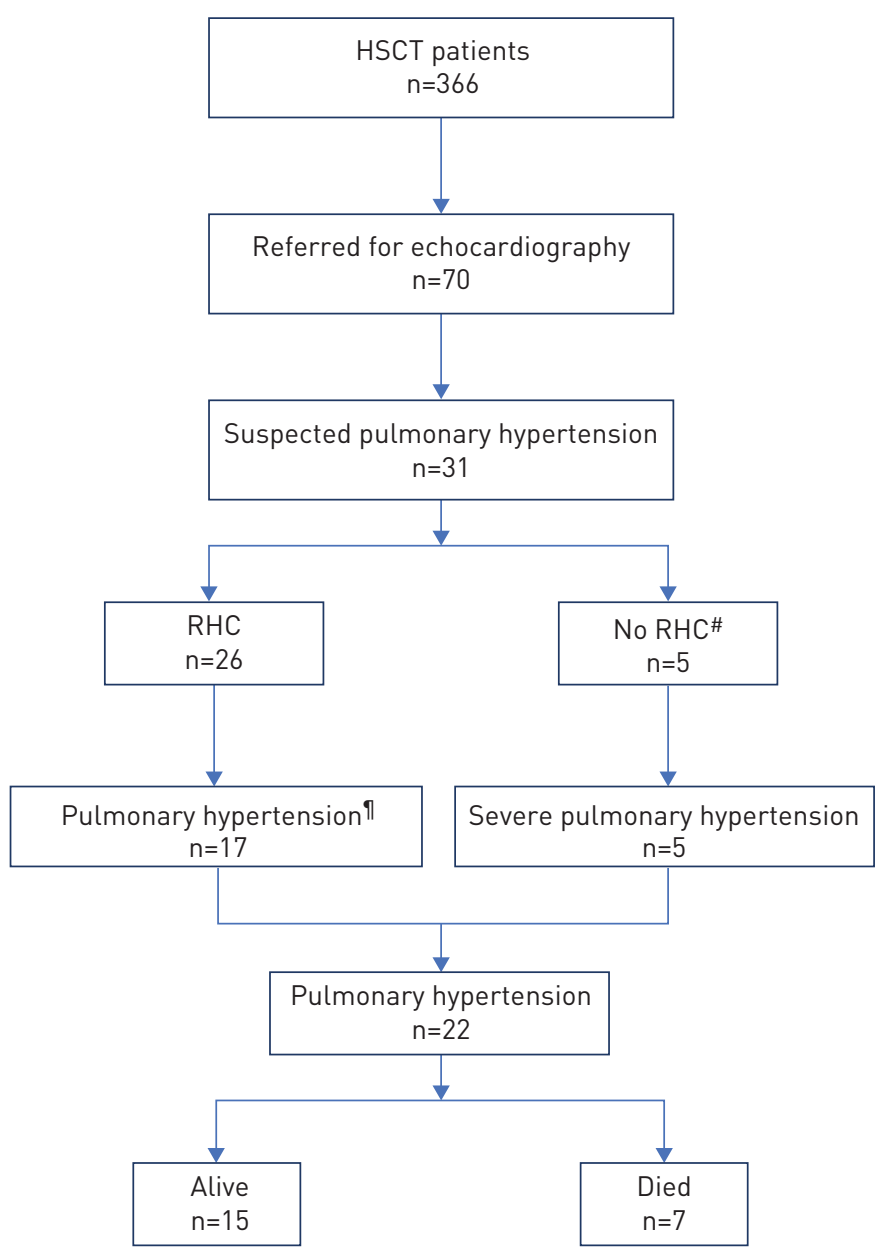

\section{Discussion}

We describe a series of 22 patients who developed severe pulmonary hypertension after HSCT with a fatal outcome in $32 \%$. Pulmonary hypertension was always revealed by respiratory distress and oxygen dependency. All the patients who died had delayed diagnosis, whereas pulmonary hypertension always resolved in those who had a rapid diagnosis allowing timely administration of targeted therapy.

Pulmonary hypertension is a rare but life-threatening complication of HSCT with a fatal evolution in the absence of a specific and rapid treatment. Dandoy et al. [2] found a mortality rate of $55 \%$, with $86 \%$ of deaths attributed to pulmonary hypertension and $14 \%$ due to relapse of the primary malignancy. In a more recent paediatric series, JODELE et al. [1] reported a lower incidence of pulmonary hypertension of $2.4 \%$ (five out of 209), but with $80 \%$ pulmonary hypertension-associated mortality.

The initial respiratory symptoms and signs associated with pulmonary hypertension after HSCT are not specific, and may also be associated to infection, bronchiolitis, interstitial pneumonitis and other HSCT-related complications. These symptoms are often misleading and lead to a delay in diagnosis, especially when pulmonary pressure is only mildly increased. Technical difficulties can lead to an incomplete echocardiography evaluation that may be initially considered normal if accurate measurements of TRV cannot be obtained. At the beginning of our series, echocardiography evaluations were not sufficiently repeated and pulmonary hypertension was diagnosed either when the clinical status severely deteriorated and/or pulmonary pressure increased to such a high level that the echocardiography could not miss the diagnosis. At that stage cardiac catheterisation was not always possible. Of note, most patients who had reactivity testing were responders. However, only one patient was efficiently treated with CCBs, whereas 12 out of 13 patients needed add-on therapy, with one death. This lack of efficacy of CCBs in responders is totally different of what is observed in idiopathic pulmonary hypertension, supporting the fact that the physiopathology of HSCT-related pulmonary hypertension differs from other causes of pulmonary hypertension.

Prior to 2012, treatment was conducted with a sequential approach starting with monotherapy, switching to bitherapy only in the absence of improvement. This management did not allow reversing the 
a)

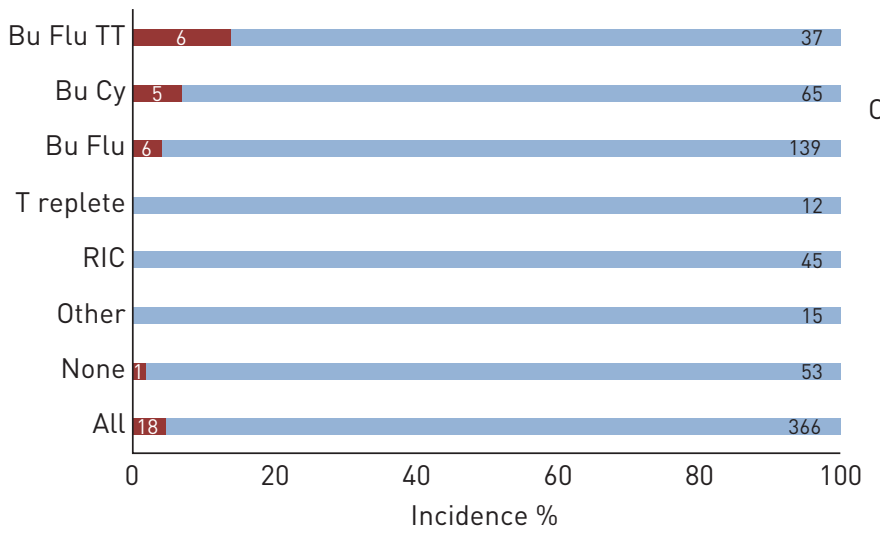

b)

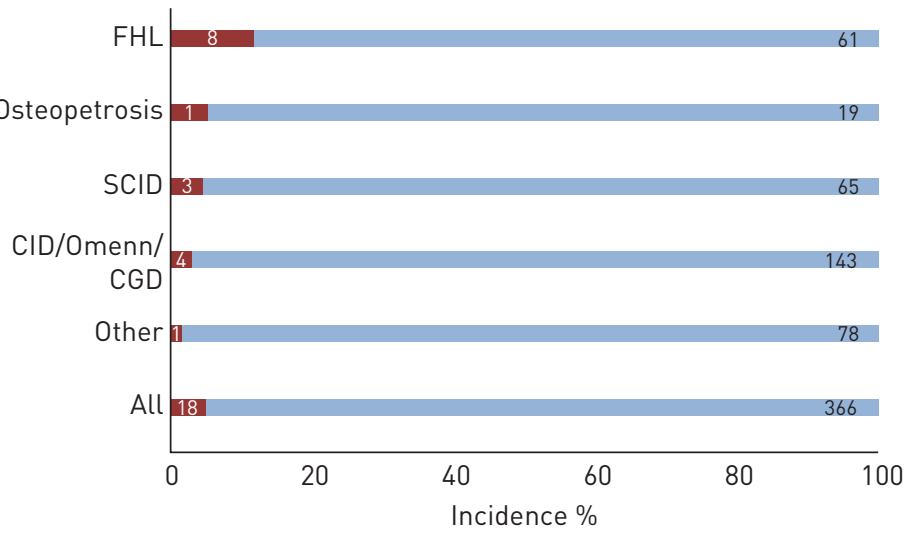

FIGURE 3 Incidence of pulmonary hypertension post-haematopoietic stem cell transplantation (HSCT): according to a) conditioning regimen and b) underlying disease. Bu Cy: busulfan and cyclophosphamide (200 $\mathrm{mg} \cdot \mathrm{kg}^{-1}$ total dose); Bu Flu: busulfan and fludarabine; Bu Flu TT: busulfan, fludarabine and thiotepa; T replete: busulfan, fludarabine and post-HSCT cyclophosphamide (100 $\mathrm{mg} \cdot \mathrm{kg}^{-1}$ total dose); RIC: reduced intensity conditioning; FHL: familial haemophagocytic lymphohistiocytosis; SCID: severe combined immunodeficiency; CID: combined immunodeficiency; Omenn: Omenn syndrome; CGD: chronic granulomatous disease.

pulmonary hypertension process in most cases, leading to a very high mortality in our initial experience ( $>50 \%$, comparable to the outcome described in the literature).

Since 2012, we intensified the management of pulmonary hypertension after HSCT allowing the last patients of our series to be diagnosed significantly earlier. In these patients, echocardiography and cardiac catheterisation were performed as soon as respiratory symptoms occurred. Targeted pulmonary hypertension treatment was initiated on the day of pulmonary hypertension diagnosis with oral combination therapy (ERA+PDE5i), evaluation was performed systematically at $24 \mathrm{~h}$ and prostanoid treatment was initiated in the absence of significant improvement. In severely ill patients, up-front tritherapy (ERA+PDE5i+prostanoids) was immediately initiated and managed in the intensive care unit. None of those last patients seen after 2012 died, and all of them normalised their pulmonary pressure within 30-45 days and could be weaned off treatment.

Our incomplete understanding of the aetiology and pathophysiology of pulmonary hypertension in HSCT patients limits targeted noninvasive diagnostic and therapeutic options. Children and young adults with

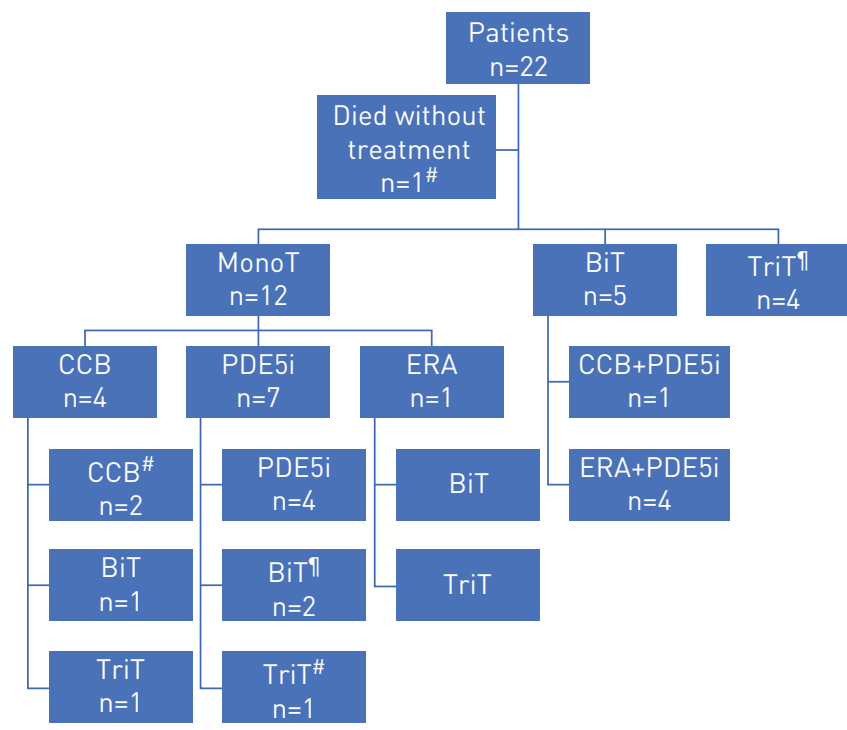

FIGURE 4 Specific pulmonary hypertension treatment administered at diagnosis and escalated in most patients. MonoT: monotherapy; BiT: biotherapy; TriT: tritherapy; CCB: calcium channel blocker; PDE5i: phosphodiesterase-5 inhibitor; ERA: endothelin receptor antagonist. \#: one death; १: two deaths. 

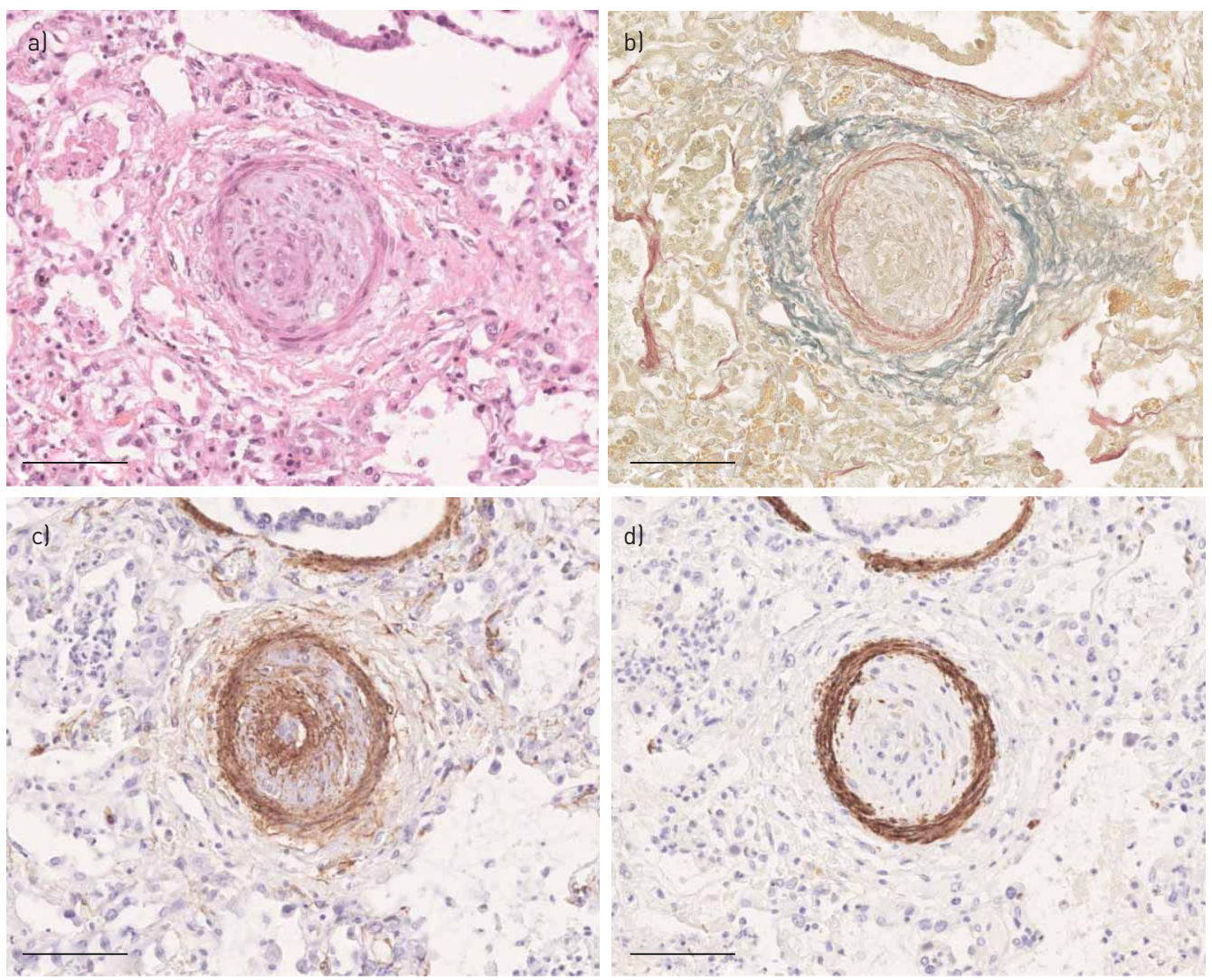

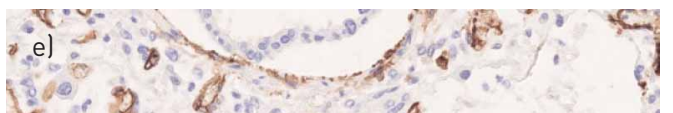
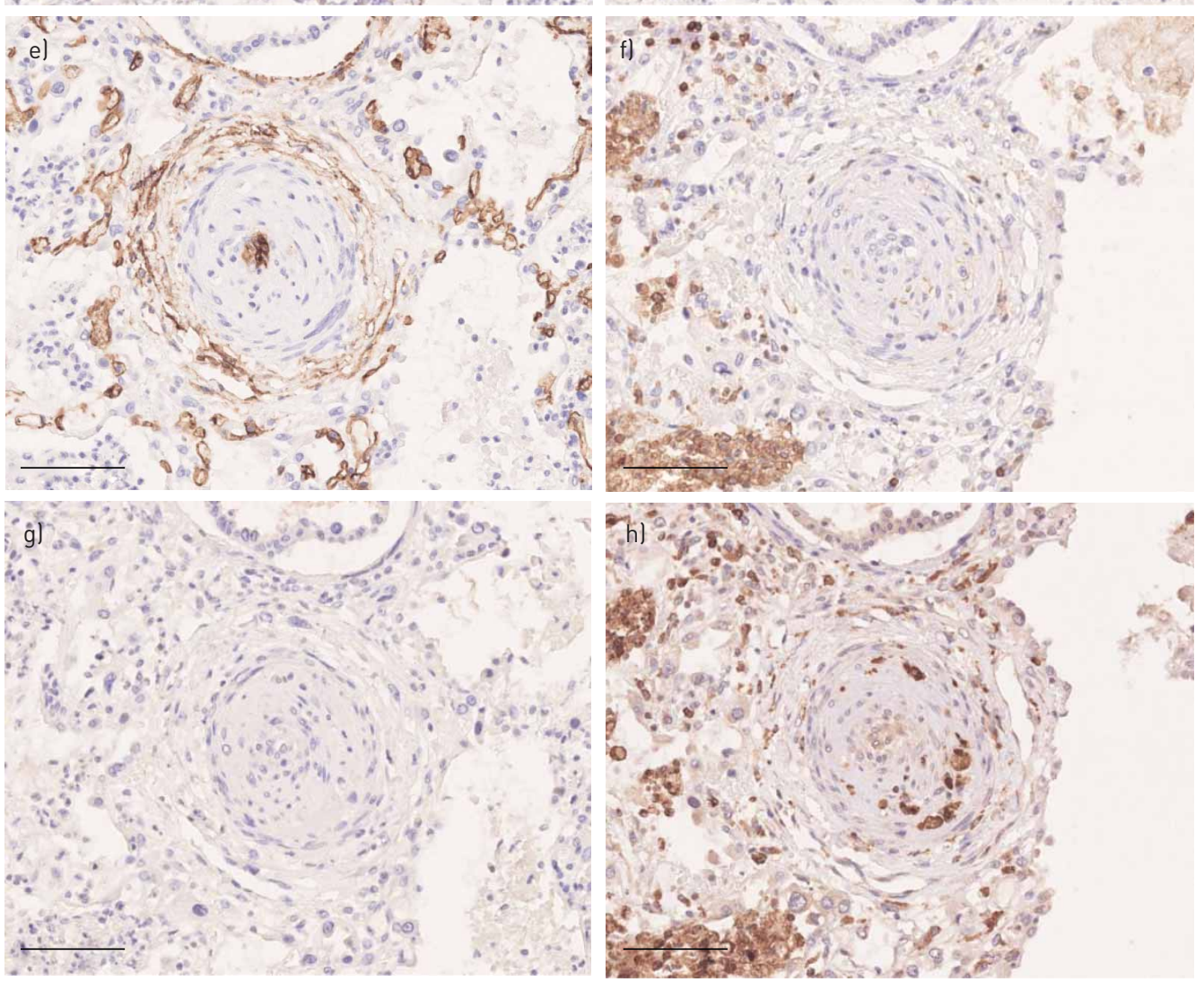

FIGURE 5 Histological lesions of intra-acinar pulmonary arteries showing intimal occlusion of the lumen by cellular proliferation. a) Haematoxylin/eosin/saffron staining, b) orcein, c) smooth muscle actin, d) caldesmon, e) CD34, f) CD45, g) CD3 and h) CD68 immunostaining indicate that intimal proliferation contains myofibroblasts, macrophages and endothelial cells, but not smooth muscle cells. Scale bar: $100 \mu \mathrm{m}$. 
pulmonary hypertension post-HSCT likely have a different mechanism of disease from idiopathic PAH or associated pulmonary hypertension. In three patients of our series, histological findings without intimal fibrosis but cellular proliferation within the pulmonary artery wall suggest an acute and potentially reversible process, and the normalisation of pulmonary pressure after aggressive treatment in our pulmonary hypertension patients is in line with this hypothesis. The intimal cellular proliferation indicates endothelial damage, supported by reports of an association with thrombotic microangiopathy as a consequence of complement activation in these patients [5]. Limsuwan et al. [7] described two patients with a reversible course of pulmonary hypertension developing 4 and 10 months after HSCT upon specific treatments.

In children undergoing HSCT, many factors could have an effect on endothelial function: drugs used for preconditioning, inflammation, infections, respiratory distress and hypoxia. In our series, all patients with respiratory symptoms were treated with antibiotics and oxygen prior to being referred to cardiology evaluation. Lung computed tomography scanning was not done in all these patients, but at least two patients had radiological lesions compatible with PVOD. However, this was not confirmed since lung biopsy performed in one of them could not find any pulmonary vein lesions.

Busulfan and cyclophosphamide have been described to cause endothelial injury [11, 12].

Defibrotide, a single-stranded polydeoxyribonucleotide with antithrombotic, anti-inflammatory and anti-ischaemic properties, has a protective effect against endothelial cell injury without increasing bleeding risks [13]. Defibrotide becomes attached to the external cell membrane of endothelial cells before being internalised by the cells, and it has been suggested that the interaction of defibrotide with the cell membrane is linked to its anti-inflammatory and antioxidant effects [14]. It has been used successfully in the treatment of hepatic veno-occlusive disease, and its prophylactic use is recommended in adults and children undergoing stem cell transplantation who present specific risk factors [15]. The effect of defibrotide with regard to the prevention and/or treatment of pulmonary hypertension in patients undergoing HSCT has not been studied in humans. In our series, more than two-thirds of the HSCT patients had received defibrotide prophylactically, notably all but two patients who developed pulmonary hypertension (including one patient who had received a nonconditioned HSCT).

Many chemotherapeutic agents have been implicated in the development of pulmonary hypertension. Dasatinib is associated with pulmonary hypertension, but none of these patients received this drug. In children with malignant disorders, previous chemotherapy also needs to be taken into account. For example, mitomycin $\mathrm{C}$ induces $\mathrm{PVOD}$ and has the characteristics of a bioreductive alkylating agent: activation of alkylating species occurs more readily under hypoxic conditions and the drug is selectively cytotoxic to hypoxic cells [16]. The underlying hypoxic condition of children who develop respiratory symptoms after HSCT may thus increase the pulmonary vascular side-effects of drug-induced pulmonary hypertension.

Inflammation and infection are very frequent in children after HSCT and have also been shown to be associated with pulmonary hypertension [17, 18], and one patient of our series developed pulmonary hypertension after HSCT despite the absence of preconditioning drugs. One study also suggests a direct effect of the bone marrow cells in the pathogenesis of pulmonary hypertension [19].

Even if the pathophysiology of pulmonary hypertension is still unclear in this population, our experience indicates that rapid treatment with oxygen and potent specific treatments of pulmonary hypertension allow improvement and pulmonary pressure normalisation in all our recent cases. Careful prospective studies, including state-of-the-art diagnostics and biomarker studies, will help elucidate mechanisms of disease and thereby help to establish optimal therapy.

In conclusion, pulmonary hypertension developing in children after HSCT is a very severe disease with a high mortality if the diagnosis is delayed. Even if respiratory symptoms are more frequently due to infection or inflammation, RHC must be performed promptly in order to detect pulmonary hypertension and to immediately initiate specific combination treatment whenever pulmonary hypertension is confirmed. Bone marrow transplant teams should seek prompt consultation with an experienced cardiologist whenever pulmonary hypertension is suspected and should insist on repeated echocardiography in case of normal values because pulmonary pressure is reactive in these children and can change dramatically from one day to another. We have shown that delayed specific treatment is very deleterious in this population. It is also important to properly communicate the pulmonary hypertension diagnosis to the anaesthesia team in any HSCT patient scheduled for procedures requiring sedatives because pulmonary hypertension may be exacerbated by such interventions, placing patients at high risk for acute decompensation. We recommend aggressive treatment strategies in this population since pulmonary hypertension is potentially reversible if diagnosed early and treated promptly. 
Acknowledgements: We particularly thank all the physicians and the nursing staff of the Depts of Paediatric Immunology, Haematology and Rheumatology of the Necker-Enfants Malades Hospital for patient care, our HSCT coordinator, Elvira Duchesne, for her excellent work, as well as the whole team of the Dept of Biotherapy of the Necker-Enfants Malades Hospital for stem cell processing.

Author contributions: M. Levy, D. Moshous and D. Bonnet: clinical care of the patients, design of the study, writing of the manuscript. I. Szezepanski: care of the patients, data collection. L. Galmiche and M. Levy: histological analysis. S. Blanche, A. Fischer, F. Lesage, L. Dupic, B. Neven and M. Castelle: critical review of the manuscript.

Conflict of interest: M. Levy has nothing to disclose. D. Moshous has nothing to disclose. I. Szezepanski has nothing to disclose. L. Galmiche has nothing to disclose. M. Castelle has nothing to disclose. F. Lesage has nothing to disclose L. Dupic has nothing to disclose. B. Neven has nothing to disclose. A. Fischer has nothing to disclose. S. Blanche has nothing to disclose. D. Bonnet reports personal fees from Actelion, Pfizer, Novartis and Bayer, outside the submitted work

\section{References}

1 Jodele S, Hirsch R, Laskin B, et al. Pulmonary arterial hypertension in pediatric patients with hematopoietic stem cell transplant-associated thrombotic microangiopathy. Biol Blood Marrow Transplant 2013; 19: 202-207.

2 Dandoy CE, Hirsch R, Chima R, et al. Pulmonary hypertension after hematopoietic stem cell transplantation. Bio Blood Marrow Transplant 2013; 19: 1546-1556.

3 Troussard X, Bernaudin JF, Cordonnier C, et al. Pulmonary veno-occlusive disease after bone marrow transplantation. Thorax 1984; 39: 956-957.

4 Steward CG, Pellier I, Mahajan A, et al. Severe pulmonary hypertension: a frequent complication of stem cell transplantation for malignant infantile osteopetrosis. Br J Haematol 2004; 124: 63-71.

5 Vaksmann G, Nelken B, Deshildre A, et al. Pulmonary arterial occlusive disease following chemotherapy and bone marrow transplantation for leukaemia. Eur J Pediatr 2002; 161: 247-249.

6 Schechter T, Leucht S, Bouffet E, et al. Pulmonary hypertensive vasculopathy following tandem autologous transplantation in pediatric patients with central nervous system tumors. Biol Blood Marrow Transplant 2013; 19: 235-239.

7 Limsuwan A, Pakakasama S, Hongeng S. Reversible course of pulmonary arterial hypertension related to bone marrow transplantation. Heart Vessels 2011; 26: 557-561.

8 Abman SH, Hansmann G, Archer SL, et al. Pediatric pulmonary hypertension guidelines from the American Heart Association and American Thoracic Society. Circulation 2015; 132: 2037-2099.

9 Douwes JM, Humpl T, Bonnet D, et al. Acute vasodilator response in pediatric pulmonary arterial hypertension: current clinical practice from the TOPP registry. J Am Coll Cardiol 2016; 67: 1312-1323.

10 Levy M, Maurey C, Celermajer DS, et al. Impaired apoptosis of pulmonary endothelial cells is associated with intimal proliferation and irreversibility of pulmonary hypertension in congenital heart disease. J Am Coll Cardiol 2007; 49: 803-810.

11 Zeng L, Yan Z, Ding S, et al. Endothelial injury, an intriguing effect of methotrexate and cyclophosphamide during hematopoietic stem cell transplantation in mice. Transplant Proc 2010; 42: 2720-2724.

12 Al-Hashmi S, Boels PJ, Zadjali F, et al. Busulphan-cyclophosphamide cause endothelial injury, remodeling of resistance arteries and enhanced expression of endothelial nitric oxide synthase. PLoS One 2012; 7: e30897.

13 Pescador R, Capuzzi L, Mantovani M, et al. Defibrotide: properties and clinical use of an old/new drug. Vascul Pharmacol 2013; 59: 1-10.

14 Palomo M, Mir E, Rovira M, et al. What is going on between defibrotide and endothelial cells? Snapshots reveal the hot spots of their romance. Blood 2016; 127: 1719-1727.

15 Dignan FL, Wynn RF, Hadzic N, et al. BCSH/BSBMT guideline: diagnosis and management of veno-occlusive disease (sinusoidal obstruction syndrome) following haematopoietic stem cell transplantation. Br J Haematol 2013; 163: 444-457.

16 Perros F, Günther S, Ranchoux B, et al. Mitomycin-induced pulmonary veno-occlusive disease: evidence from human disease and animal models. Circulation 2015; 132: 834-834.

17 Duncan M, Wagner BD, Murray K, et al. Circulating cytokines and growth factors in pediatric pulmonary hypertension. Mediators Inflamm 2012; 2012: 143428.

18 Smadja DM, Gaussem P, Mauge L, et al. Comparison of endothelial biomarkers according to reversibility of pulmonary hypertension secondary to congenital heart disease. Pediatr Cardiol 2010; 31: 657-662.

19 Yan L, Chen X, Talati M, et al. Bone marrow-derived cells contribute to the pathogenesis of pulmonary arterial hypertension. Am J Respir Crit Care Med 2016; 193: 898-909. 